\title{
Medical Oxygen: A Lifesaving Drug During the COVID-19 Pandemic - Source and Distribution
}

\author{
Javed Ismail ${ }^{1} \cdot$ Arun Bansal $^{2}$ (1) \\ Received: 16 May 2021 / Accepted: 19 August 2021 / Published online: 14 January 2022 \\ (c) Dr. K C Chaudhuri Foundation 2021
}

\begin{abstract}
Oxygen is an essential lifesaving medicine used for several indications at all levels of health care. The COVID-19 pandemic and its recent second wave have resulted in a surge in demand for necessary resources, including trained staff, hospital beds, and medical supplies like oxygen. Limited availability of these resources resulted in added risk of adverse outcomes. Also, the widespread unregulated use of oxygen by the general public in household settings poses safety concerns. This review focuses on sources of medical oxygen like cryogenic oxygen plants, pressure swing adsorption, oxygen concentrators, and oxygen cylinders. Their specifications, storage, distribution within healthcare settings, regulation, and safety concerns have been considered. Resources needed for calculating oxygen demand, surge planning, identifying the suitable source, and distribution systems for different settings have been detailed. This review aims to help the hospital administrators, biomedical engineers, and clinicians plan and rationalize oxygen usage in low- and middle-income countries during the COVID-19 pandemic.
\end{abstract}

Keywords Medical oxygen · Cryogenic distillation · Pressure swing adsorption · Oxygen concentrators · Oxygen cylinders · COVID-19

\section{Introduction}

Medical oxygen is a widely used life support therapy in various diseases at all levels of health care. Oxygen is also used in multiple industries like glass, metallurgy, chemical, wastewater treatment, and fish farming. Initial reports from China suggested that about $15 \%$ of patients with COVID19 had a severe illness requiring oxygen, and $5 \%$ needed intensive care [1]. Hypoxemia due to COVID-19 pneumonia is a life-threatening complication and requires oxygen supplementation for treatment. During the second wave of the COVID-19 pandemic in India, oxygen demand has increased exponentially, resulting in the 'Oxygen Crisis.' In India, the

The study was carried out at Postgraduate Institute of Medical Education and Research (PGIMER), Chandigarh, India.

Arun Bansal

drarunbansal@gmail.com

1 Pediatric Intensive Care Unit, NMC Royal Hospital, Abu Dhabi, United Arab Emirates

2 Pediatric Emergency and Critical Care Unit, Department of Pediatrics, Advanced Pediatrics Center, Postgraduate Institute of Medical Education and Research (PGIMER), Chandigarh 160012, India
pre-COVID need for medical oxygen was about 700 metric tons (MT) per day. The demand surged to 3,100 MT per day during the first wave and 8900 MT, as of $6^{\text {th }}$ May 2021, during the second wave [2]. To meet this demand, the production was increased to 9400 MT as of $6^{\text {th }}$ May 2021 [2]. Given the rapidly increasing cumulative active cases (3.7 million, first week of May 2021), estimates project a much higher oxygen demand of 11-19 thousand MT per day [3, 4]. The Government of India diverted almost all the industrial oxygen for medical use, hoping that the total production can meet hospital demand. However, a relative shortage of cylinders and specialized tankers for storage and transport has precipitated this crisis further.

Medical oxygen falls under the ambit of Sect. 3(b)(i) of the Drugs and Cosmetics Act, 1940 and the central government can regulate its manufacturing, distribution in the public interest. The licensure for manufacturing medical oxygen is issued by 'The Drug Controller General of India'. The Gas cylinder rules, 2004 regulate the ownership, filling and transport of compressed oxygen in cylinders.

Medical oxygen is a drug that should be prescribed by a physician and the therapy be optimized as indicated in an appropriate setting. Like any other drug, medical oxygen exhibits toxic effects if used in doses higher than the body's 
demand. Often it can occur in an unregulated or unmonitored settings, resulting in hyperoxia and signs of oxygen toxicity. Therefore, the importance of optimal oxygen therapy cannot be overstated. This review focuses on sources of medical oxygen, its distribution, regulation, and specifications related to it.

Learning objectives:

- Principles of production, storage, transport and distribution of medical oxygen

- Safety concerns relating to medical oxygen use

- Planning of oxygen demand for healthcare facilities

\section{Sources of Medical Oxygen}

Medical grade oxygen should have at least $82 \%$ of pure oxygen. The choice of source of oxygen depends on several factors, including location, infrastructure, cost, availability of electricity, resources for maintenance and spares, etc. (Table 1).

\section{Cryogenic Distillation/Liquid Oxygen Plant}

Principle of oxygen production: Large-scale commercial production of oxygen involves cryogenic liquefaction and fractional distillation of air. This process was first used on a large scale by Von Linde and Hampson in 1895. The steps involved are filtration of air to remove impurities, compression, and liquefaction by cooling. Carbon dioxide, which liquefies at $-80{ }^{\circ} \mathrm{C}$, is removed, followed by further cooling to $-200{ }^{\circ} \mathrm{C}$. This cooled liquefied carbon dioxide-free air is passed through the fractioning column's warmer bottom $\left(-183^{\circ} \mathrm{C}\right)$. Nitrogen boils at $-195^{\circ} \mathrm{C}$, exits the column leaving behind liquid oxygen, which is further purified to remove argon (Fig. 1).

Storage and transport: This oxygen is a pale blue liquid of high purity (about $99 \%$ ) cooled to $-183{ }^{\circ} \mathrm{C}$. It needs specialized cryogenic tanks for transport and storage (Fig. 2a-d). Hospitals store the liquid oxygen in cryogenic tanks and can supply it to the piped system by self-vaporization. These tanks need periodic refilling from commercial liquid oxygen plants. As commercial plants are located far off from medical facilities and the number of cryogenic tankers is limited, transport was a critical determinant of oxygen delivery to hospitals during the second wave of COVID-19. As of the first week of May 2021, there were only 1,681 tankers with a total capacity of 23,056 MT, which is grossly inadequate [2].

Electrical supply: Since liquid oxygen is delivered by self-vaporization through the piped system, it does not require an electrical supply.

Specifications: Cryogenic tanks, also called Vacuum Insulated Evaporator (VIE) tanks, are vacuum insulated, double-walled, and consist of two concentric vessels. The outer vessel is made of carbon steel, and the inner vessel of austenitic steel. The space between two vessels is

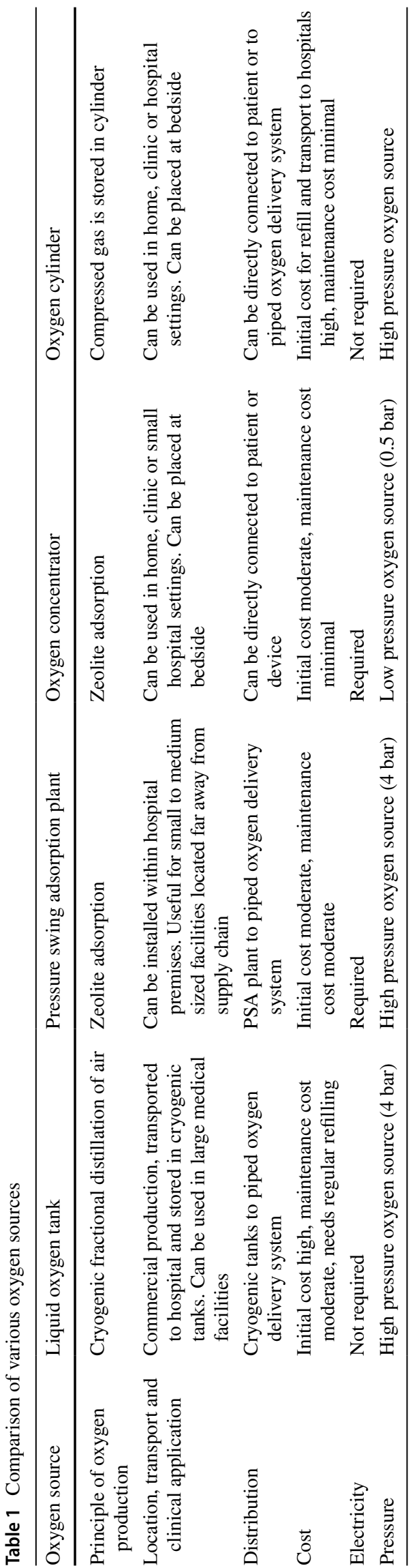




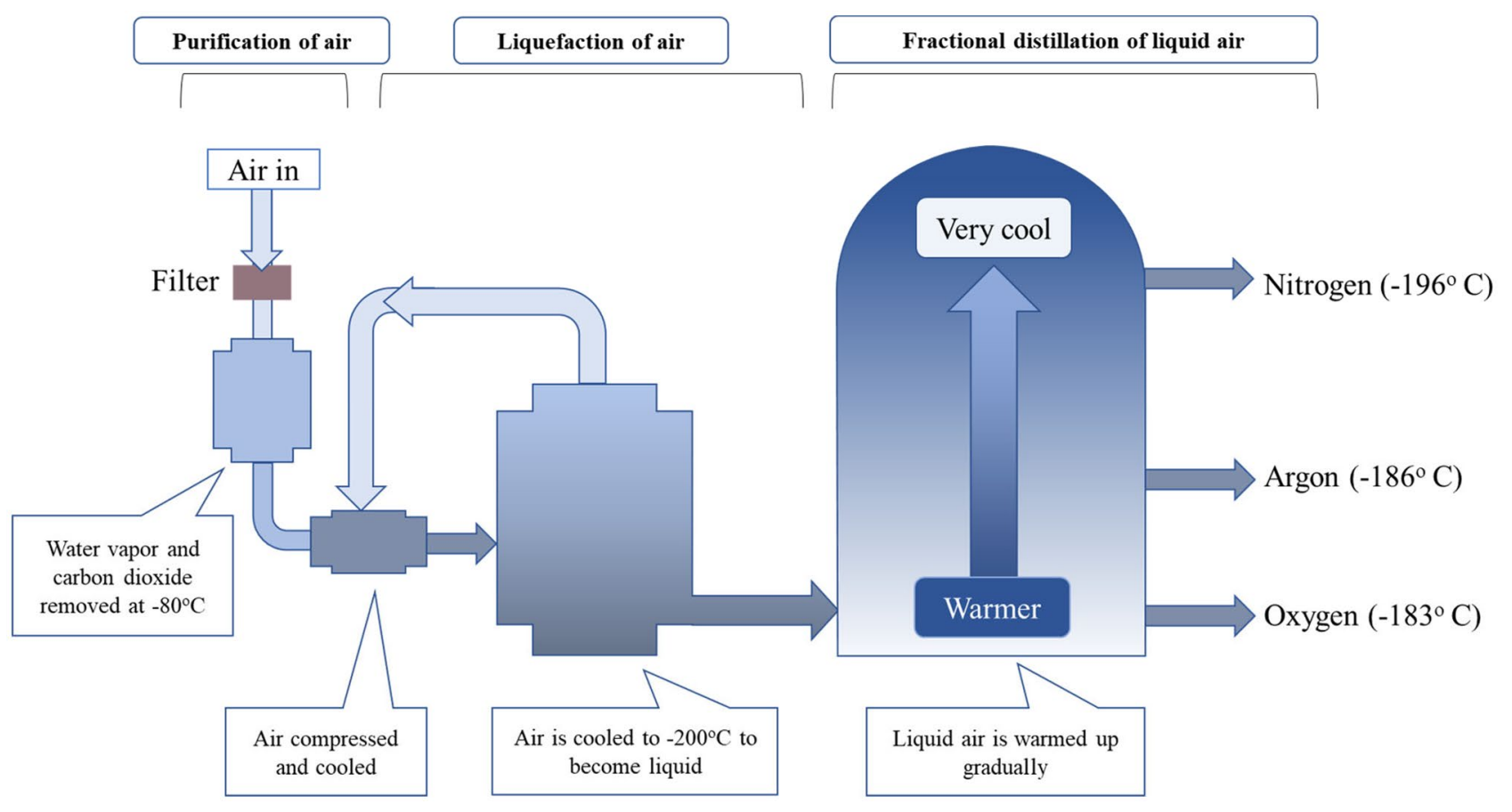

Fig. 1 Illustration of cryogenic fractional distillation of air to produce medical oxygen

evacuated and filled with perlite. The tank is connected to an evaporator converting liquid oxygen to gaseous form before being distributed through the pipeline. All tanks have vertical configuration and require a limited space for installation (about $15 \mathrm{~m} \times 9 \mathrm{~m}$ ). The gross capacities of tanks range from 3160 to $61,600 \mathrm{~L}$, thus suited for large hospitals. The maximum allowable working pressure is 18-36 bar. These are routinely tested per internationally accepted standards.

Advantages and disadvantages: Compared to compressed gas from oxygen cylinders, the purity is high, pressure delivered is stable, chances of contamination are nil, and handling and maintenance are easy. However, the operating cost is high and needs regular refilling.

Safety: In case of system leakage, the safety valves blow off, and the oxygen is released into the atmosphere.

\section{Pressure Swing Adsorption (PSA) Plant}

Pressure swing adsorption units are being used as a source of medical oxygen since 1970 . They can be installed in hospitals and are a reliable, economical source.

Principle of oxygen production: These units have two zeolite sieve filled towers that adsorb nitrogen from the compressed atmospheric air. Since oxygen is not adsorbed by zeolite, it gets separated and is stored in buffer tanks (Fig. 3a, b). These towers work alternatively to generate oxygen, i.e., while the first tower adsorbs nitrogen and separates oxygen from the air, the second tower regenerates by releasing adsorbed nitrogen to the atmosphere. Oxygen produced by this method is of steady purity, $93 \pm 3 \%$, as per the Indian Pharmacopeia 2010 guidelines. Oxygen output is delivered to the piped system at an optimized pressure of 4 bar.

Electrical supply: They require an uninterrupted, reliable power supply with voltage stabilization.

Specifications: The capacity of these generators ranges from 5-200 $\mathrm{m}^{3}$ per hour and can function round the clock. During the first wave of the COVID-19 pandemic, the Indian government commissioned the installation of 162 PSA plants. In May 2021, it expanded the total demand to 1594 PSA plants for medical use [2].

Safety: A digital analyzer monitors oxygen purity before it goes to the storage tank. An alarm is activated if oxygen purity falls below $90 \%$ or pressure below 3.5 bar in the tank, and the oxygen supply is auto switched to a reserve source.

Advantages and disadvantages: It is economical and can be used to fill oxygen cylinders. The space needed for installation is small and can be installed within the hospital premises. It requires a continuous, uninterrupted electrical supply.

\section{Oxygen Concentrators}

Oxygen concentrators are compact electrical devices used at home or small clinics to generate oxygen from atmospheric air. They are a preferred source of home oxygen for patients on long-term continuous oxygen therapy. 
Fig. 2 a Liquid oxygen for transport in cryogenic tankers, b Cryogenic tanks for liquid oxygen storage in industry and, c hospital use, $\mathbf{d}$ Control panel for liquid oxygen plant
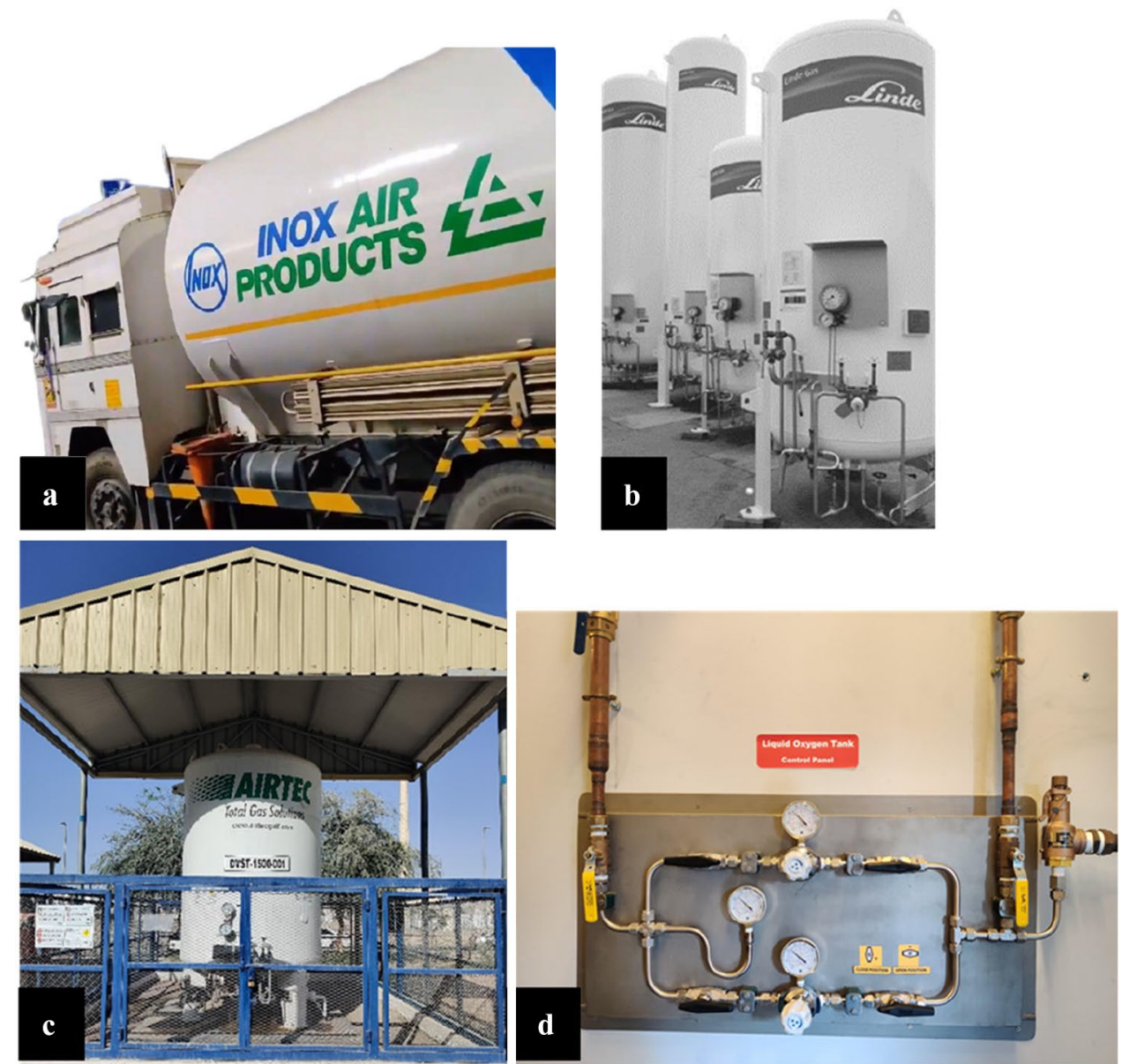

Principle of oxygen production: Concentrators use PSA technology with zeolite adsorption from ambient air and concentrating oxygen to more than $90 \%$ purity (Fig. $4 a, b$ ).

\section{Specifications:}

- Flow rate and oxygen concentration: Oxygen delivery rates range from $1-10 \mathrm{~L} / \mathrm{min}$. Most devices generate oxygen at a purity of $>93 \%$. However, some concentrators deliver a variable purity, i.e., as the flow rate increases, the purity of the oxygen decreases. Thus, the purity at the highest flow rate is an important factor to be considered while procuring a concentrator. Concentrators that can deliver higher flow rates $(10 \mathrm{~L} / \mathrm{min})$ have a larger compressor and are heavier (above $20 \mathrm{~kg}$ ). Critical COVID-19 patients may require high flow rates $(10 \mathrm{~L} / \mathrm{min})$ with constant purity of $90 \%$. So, the devices with variable purity or lower flow rates are not suitable for managing such patients [5].

- Pulse flow or constant flow: Concentrators usually have two flow modes: constant flow (most common) or pulse flow.
In the pulse flow mode, the machine senses the patient's inspiratory effort and delivers oxygen during inspiration. Pulse flow devices are often small, compact, suited for ambulatory patients with low oxygen requirements. But they cannot be connected to other ventilation devices like CPAP, BiPAP. Constant flow is preferred for patients requiring a flow rate above $4 \mathrm{~L} / \mathrm{min}$ and can be connected to ventilation devices.

Electrical supply: They require an uninterrupted, voltage stabilized electrical power supply. Smaller battery-operated portable devices are available for ambulatory patients. Spare batteries should be kept charged and ready for backup.

Safety: Concentrators have safety alarms to detect low oxygen concentration, no flow, power supply failure, and high temperature. Regular maintenance should be done to check the functioning of the zeolite sieve and may need periodic replacement every $25,000 \mathrm{~h}$. Some concentrators have an inbuilt digital display of oxygen purity and flow rate. Environmental conditions like a temperature between $0-40{ }^{\circ} \mathrm{C}$ and relative humidity of $15 \%-95 \%$ are required for optimal functioning. Noise levels of $<50 \mathrm{~dB}$ are ideal for domestic use. 
Fig. 3 a Illustration of pressure swing adsorption plant, b PSA plant installed in a hospital
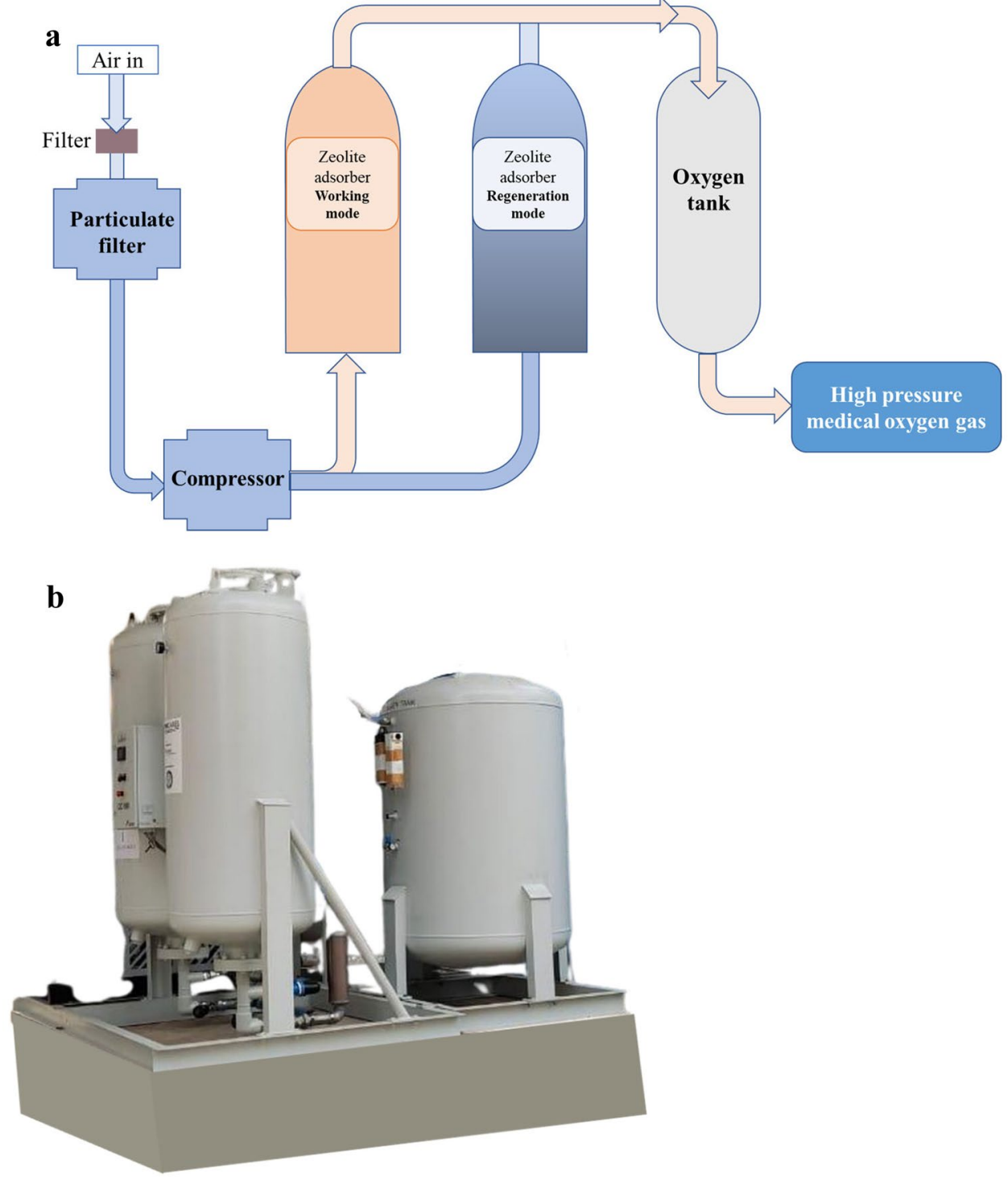

Instructions for safe home use:

- Switch off the concentrator when not in use

- Do not leave oxygen tubing on the bed

- Place concentrator at least $30 \mathrm{~cm}$ away from the wall

- Clean the filters regularly (every week or more frequently if used in a dusty environment)

- Replace the sterile water in the humidifier at regular intervals

- Keep away from fire or flammable objects

- Regular maintenance should be done every 5,000 h or annually

- Keep the surface clean, report if any signs of damage

- Familiarize with different alarms before use
Advantages and disadvantages: These devices are compact, portable, and easy to use. They are suitable for home use. However, they need a reliable electrical supply; oxygen generated is of low pressure ( 0.55 bar) and cannot be used with high-pressure devices like ventilators or oxygen blenders.

\section{Oxygen Cylinders}

Principle: Oxygen generated at industrial cryogenic or PSA plants is filled into high-pressure cylinders in compressed gas form. They are available for easy storage, transport, and used in various settings like homes, hospitals, and ambulances. 
Fig. 4 a Illustration of oxygen concentrator circuit [5], b Commonly available oxygen concentrator devices
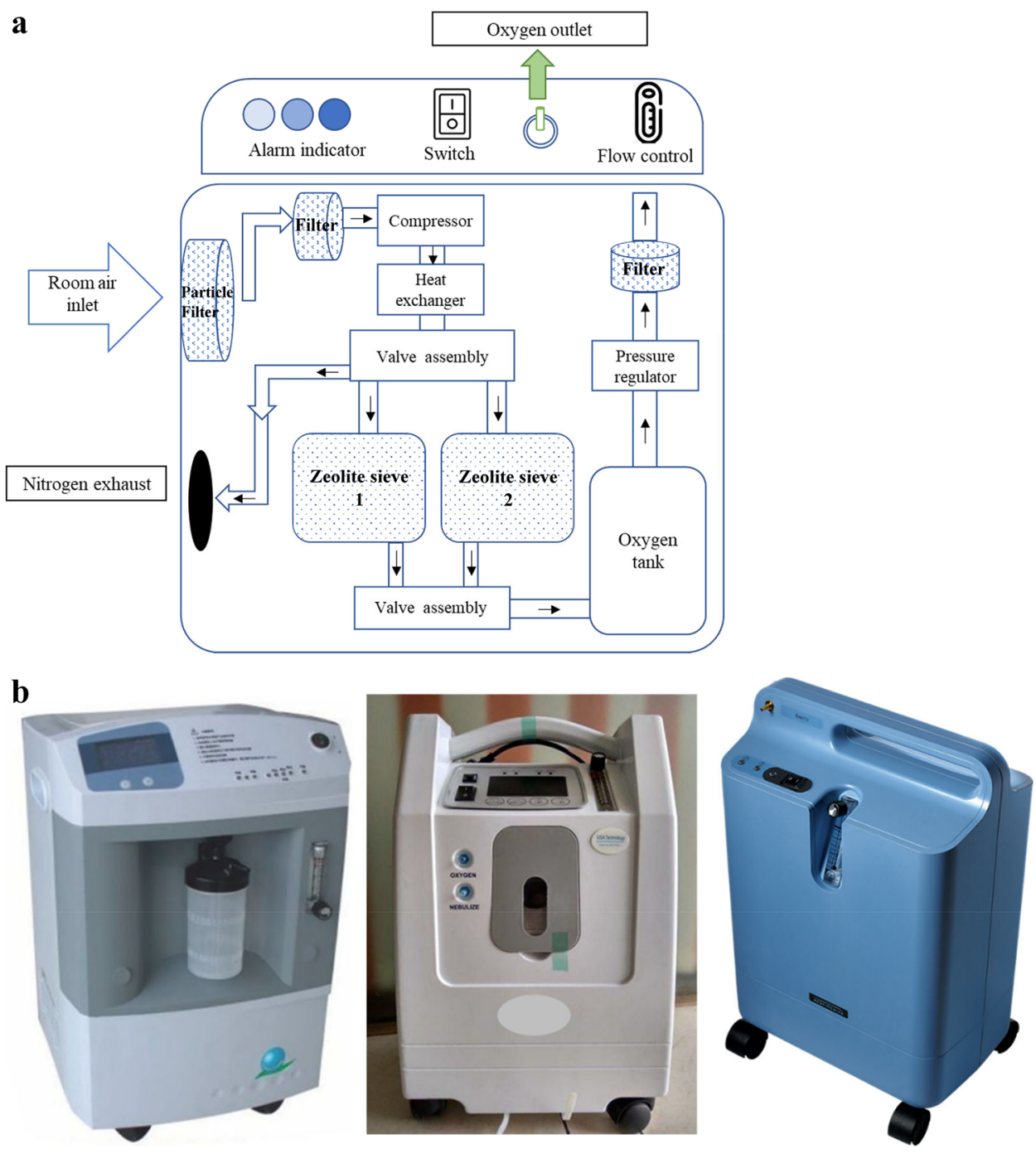

Specifications: Cylinders are usually made of steel alloys. MRI compatible are lightweight aluminum cylinders. The valve on the top of the cylinder controls the outflow of oxygen, and the pressure gauge indicates the degree of gas fill. Labels on the cylinder's shoulder displays various details like name, chemical symbol, diamond-shaped hazard warning, tare weight, volume, manufacturer name, safety instructions, and emergency contact details. The engraving contains various information like cylinder content, test pressure, and date of test performed. Valves used to connect the cylinder are usually two types: pin index valve and bull-nose valve. A regulator with flow meter assembly threaded to the valve allows the cylinder to be connected to the patient.

Oxygen cylinders are color-coded as white (ISO coding) or green (US coding). They are available in various dimensions and storage capacities, coded as B, D, E, G, and J (Fig. 5). All cylinder sizes, when filled, are under a pressure of 200 bar. However, the normal filling pressure is $137 \mathrm{bar}$ (or 2000 psi approx.). If cylinder gauge pressure and cylinder capacity are known, the volume of oxygen in the cylinder can be calculated using the formula:

The volume of oxygen in a cylinder $(\mathrm{L})=$ Gauge pressure (bar) x Cylinder volume (L).

The approximate number of hours a cylinder will last can be calculated if the flow rate required for the patient is known, using the given formula:

Time duration his cylinder would last (in minutes) $=\mathrm{Oxy}$ gen volume $(\mathrm{L}) \div$ Flow rate $(\mathrm{L} / \mathrm{min})$

OR

$=[$ Gauge pressure $\mathrm{x}$ Cylinder volume $] \div$ Flow $(\mathrm{L} / \mathrm{min})$

For example, a patient ' $\mathrm{X}$ ' requires a flow of $4 \mathrm{~L} / \mathrm{min}$ and has a cylinder with gauge pressure 100 bar in an $\mathrm{E}$ type cylinder $(4.7 \mathrm{~L})$.

The volume of oxygen in a cylinder $(\mathrm{L})=$ Gauge pressure (bar) x Cylinder volume (L)

$$
=100 \times 4.7=470 \mathrm{~L}
$$


Fig. 5 Oxygen cylinders of different capacities-as compressed gas in high pressure cylinders

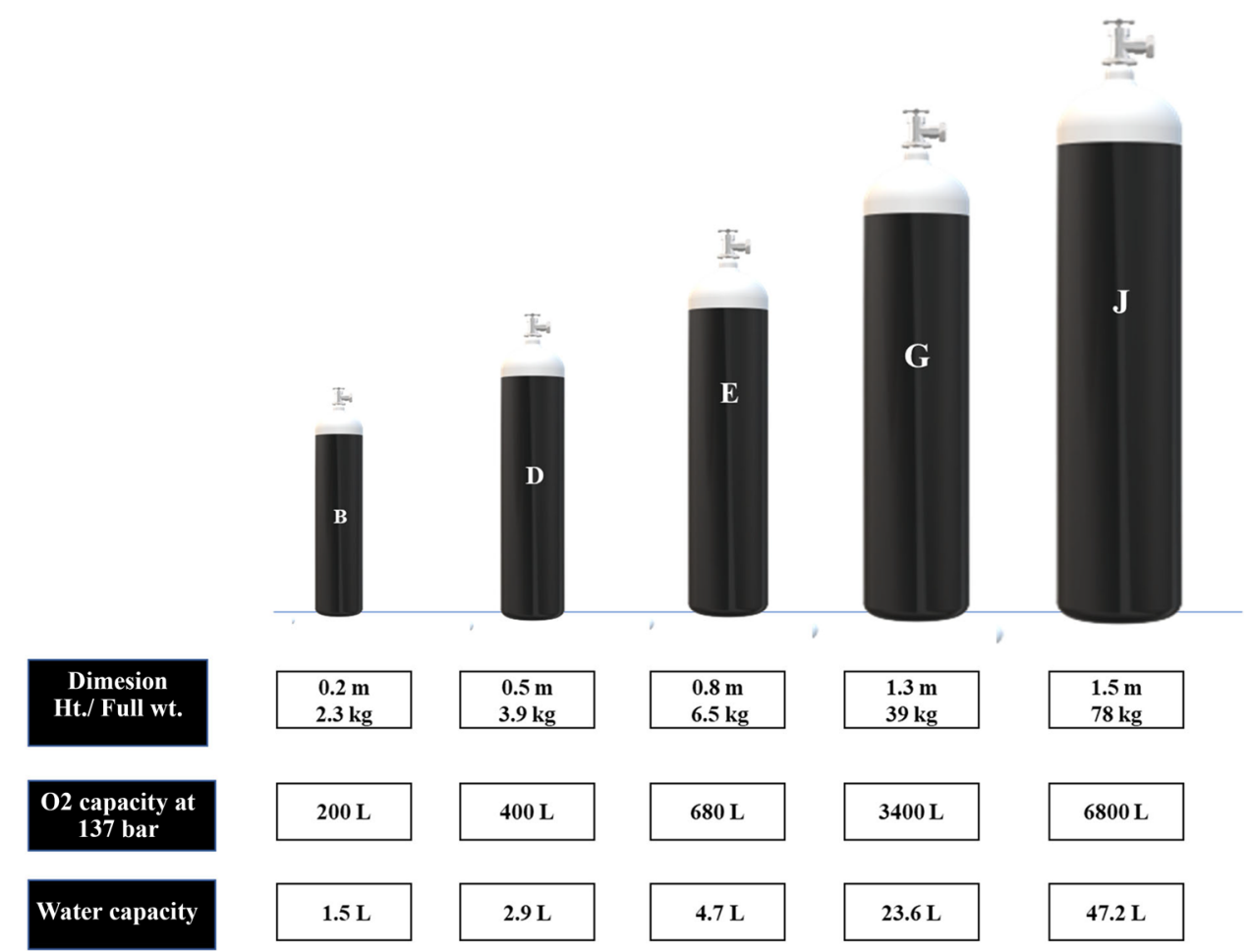

supply; distribution is labor-intensive and dependent on suppliers and transport.

\section{Distribution and Regulation of Medical Oxygen within the Hospital}

Medical oxygen goes through the manifold from the primary source and is distributed through piped medical gas and vacuum (PMGV) system. The manifold room is set up away from the main hospital building and accessible to the delivery vehicles. The room should be temperature controlled at $10-40{ }^{\circ} \mathrm{C}$, well ventilated, and should have necessary instructions and warning signs. Manifold also stores two banks of oxygen cylinders as a secondary source; each should last at least $2 \mathrm{~d}$ based on average weekly demand. They remain connected to the PMGV system via copper tailpipes. A separate reserve of cylinders that can sustain for $3 \mathrm{~d}$ is also stored appropriately in the manifold room. The manifold should always have an adequate reserve of oxygen cylinders to sustain $1 \mathrm{wk}$.

Oxygen pipelines of the PMGV system are made of specialized deoxidized copper alloy, valves, gauges, outlets, and alarms. The size of the pipe decreases from $42 \mathrm{~mm}$ in the manifold to $15 \mathrm{~mm}$ at the patient outlet. Oxygen pipes are color-coded as white from the manifold till it reaches the patient outlet. Oxygen delivery is divided in the wards into zones controlled by zone valves (also called area valve service unit), facilitating isolation of a particular area for repair Advantages and disadvantages: Easy to use, serves as a
secondary and/or reserve source. Limitations are exhaustible 
and maintenance. The ISO standard, ISO 7361-1:2007 details about the installation, maintenance and operation of medical gas distribution systems.

Alarm system: The alarm system in the pipeline distribution operates at three different levels: master alarms, zone alarms, and area alarms. Master alarms monitor manifold and oxygen source; zone alarms monitor defined zones, typically a floor or part of it; area alarms operate to a specific area within the zone. Each alarm is associated with a pressure gauge and valve that can be used to isolate a part of the pipeline for maintenance. Standard oxygen distribution alarms are high pressure, low pressure, reserve in use, reserve low pressure, and reserve less than average day's supply. Regular maintenance of valves, gauges, and alarms should be carried out.

A particular facility should have a primary, secondary, and reserve supply of oxygen. Each supply system can be a combination of any of the following: cryogenic liquid oxygen tanks, PSA plants, cylinders, or concentrators. Primary and secondary supply remain connected to the oxygen supply pipeline system. Reserve supply is connected to a specific section of the pipeline and can be used in the event of failure of both primary and secondary supplies. Oxygen cylinders are usually restricted for secondary or reserve supply.

Oxygen outlet: At the patient end, oxygen is received at the wall outlet, and its flow is regulated with flow meters. Oxygen outlets are color-coded as white, clearly labeled, and self-sealing, automatically shutting down as the Schrader probes are withdrawn. The color-coded flexible hoses (white or green) connect the outlet with the devices, and their fittings are pin-indexed to match each other mechanically, to prevent connection errors. The risk of oxygen hose rupture during transport is high. Regular maintenance and replacement of the hoses every $2-5 \mathrm{y}$ are recommended.

Flow meters: Flow meters are usually three types; Thorpe tube, Bourdon gauge, and dial/click type. Commonly available are the Thorpe tube flow meters, which can be used in wall outlets and cylinders. Bourdon gauge and dial/click type are suited for cylinders. Thorpe tubes have transparent, graduated columns calibrated to the patient inlet pressure range of 3.8-4 bar. They are available in wide flow ranges, are simple and cost-effective to use. The disadvantage is that it works only in a vertical position and is fragile.

Oxygen flow meters are equipped with non-heated bubble humidifiers to provide humidified oxygen to the patient. These are connected between the oxygen port and the patient breathing circuit. Sterile water in the humidification bottle should be changed at regular intervals to prevent contamination.

\section{Calculating the Oxygen Requirement for the Hospital}

India's Ministry of Health and Family Welfare has fixed the maximum limit for oxygen supply to $40 \mathrm{~L} / \mathrm{min}$ for an intensive care bed and $15 \mathrm{~L} / \mathrm{min}$ for a regular bed to limit the wastage [4]. Using the above criteria, oxygen requirements for a particular facility can be calculated. For example, for a 100 bedded facility with 20 ICU beds and 80 regular beds,

Total oxygen required $=($ No. of beds $x$ Flow rate for ICU beds $)+($ No. of beds $x$ Flow rate for regular beds $)=(20 \times 40$ $\mathrm{L} / \min \times 60)+(80 \times 15 \mathrm{~L} / \min \times 60)=120,000 \mathrm{~L} / \mathrm{h}$ or 120 $\mathrm{m}^{3} / \mathrm{h}$.

Accordingly, the facility can install a PSA or liquid oxygen storage tank $(1 \mathrm{~L}$ of liquid oxygen $=861 \mathrm{~L}$ of oxygen gas). Online open-source calculators are available (https:// fervent-carson-c81c94.netlify.app/supply).

The surge in demand for oxygen during COVID-19 needs planning at various levels, from the government to the individual facilities. It should cover various aspects like estimating demand, production, diverting industrial output, storage, transport, distribution, and logistics involved in each of these steps. Lead time for implementing the oxygen surge plan should be factored while planning. World Health Organization has developed a surge calculator tool for COVID-19 to estimate the demand during the surge $[6,7]$.

\section{Oxygen Delivery Devices}

For an individual patient, the commonly used oxygen delivery devices are.

- Nasal cannula: 1-2 L/min in children; $3-5 \mathrm{~L} / \mathrm{min}$ in adults

- Venturi mask: 6-10 L/min

- Non-rebreathing mask: $10-15 \mathrm{~L} / \mathrm{min}$

- For high flow nasal cannula, noninvasive ventilation, or invasive ventilation, oxygen flow rates vary according to the total gas flow rate and the set oxygen. These devices typically require high pressure (4 bar) and high flow rates.

\section{Conclusion}

Medical oxygen has become a critical lifesaving resource during the COVID-19 pandemic. Detailed knowledge of its production, storage, and distribution to reach the bedside might help plan to overcome the oxygen crisis. Its judicious use and standard measures of safety can prevent wastage and hazards related to this critical resource. 
Authors' Contributions Study concept and design: JI and AB; Critical revision of the manuscript for important intellectual content: JI and AB; Administrative, technical, or material support: JI and AB; Study supervision: $\mathrm{JI}$ and $\mathrm{AB}$. $\mathrm{AB}$ had full access to all the data in the study and took responsibility for the integrity of the data and the accuracy of the data analysis. $\mathrm{AB}$ will act as the guarantor for this paper.

\section{Declarations}

\section{Conflict of Interest None.}

\section{References}

1. Wu Z, McGoogan JM. Characteristics of and important lessons from the coronavirus disease 2019 (COVID-19) outbreak in China: summary of a report of 72314 cases from the Chinese center for disease control and prevention. JAMA. 2020;323:1239-42.

2. Centre undertakes multiple initiatives to enhance oxygen availability, distribution and storage infrastructure. PIB, Government of India. 2021. Available at: https://pib.gov.in/Pressreleaseshare. aspx?PRID=1717459. Accessed on 11th May 2021.

3. COVID-19 Oxygen Needs Tracker. Path. 2021. Available at: https://www.path.org/programs/market-dynamics/covid-19oxygen-needs-tracker/. Accessed on 11th May 2021.

4. Kaushik M. How many more lives could be lost in India's oxygen crisis? Scroll.in. 2021. Available at: https://scroll.in/article/ 994252/how-many-more-lives-could-be-lost-in-indias-oxygencrisis. Accessed on 11th May 2021.

5. WHO technical specifications for oxygen concentrators. World Health Organization. 2015. Available at: https://www.who.int/ publications/i/item/9789241509886. Accessed on 14th May 2021.

6. Coronavirus disease (COVID-19) technical guidance: Essential resource planning. World Health Organization. 2020. Available at: https://www. who.int/emergencies/diseases/novel-coronavirus-2019/technicalguidance/covid-19-critical-items. Accessed on 14th May 2021.

7. Oxygen sources and distribution for COVID-19 treatment centres. World Health Organization. 2020. Available at: https://www.who. int/publications-detail-redirect/oxygen-sources-and-distributionfor-covid-19-treatment-centres. Accessed on 14th May 2021.

Publisher's Note Springer Nature remains neutral with regard to jurisdictional claims in published maps and institutional affiliations. 